\title{
Comparative Study of Safety and Efficacy between Propofol-Fentanyl Versus Propofol-Dexmeditomidine Combination For Sedation in Upper Gastro-Intestinal (GI) Endoscopic Procedures- A Prospective Randomised Study
}

\author{
Ajay Basarigidad ${ }^{1}$, Sandeep Killedar ${ }^{2}$ \\ ${ }^{1}$ Assistant Professor, Department of Anaesthesiology and Critical care, Gadag Institute Of Medical Science, Mallasamudra Gadag- $582103,{ }^{2}$ Senior \\ Resident Department of Anaesthesiology and Critical care, Sapthagiri Institute of Medical Sciences \& Research Centre, Chikkasandra, Hesaraghatta Main \\ Road, Bangalore - 560090
}

\section{Abstract}

Background: Endoscopy in patients with gastrointestinal disorders (GI) is of immense benefit for diagnostic and therapeutic measures. Inspite of use of flexible fibreoptic equipments, endoscopy remains an unpleasant experience for most patients. The purpose of sedation in these patients is to relieve anxiety, discomfort or pain, and diminish memory of the event. There has been a general consensus that moderate sedation provides adequate control of pain and anxiety during endoscopic procedures. Conscious sedation enables patients to maintain their response to verbal and tactile stimuli without losing cardiovascular and ventilatory function. The aim of study is to compare the safety and efficacy between propofol-fentanyl and propofol-dexmeditomidine combination for sedation in upper gastro-intestinal (GI) endoscopic procedures. Subjects and Methods: A prospective study of 70 cases of both sexes belonging to ASA Grade I,II and III. Planed for elective upper GI endoscopies under sedation were included in this study were randomly selected. The study group was divided in two groups of 35 each, Group A Propofol-Fentanyl (PF) and Group (B) Propofol-Dexmeditomedine (PD). In the PF group, patient was administered fentanyl $2 \mathrm{mcg} / \mathrm{kg}$ initially followed by Propofol loading dose of $1.5 \mathrm{mg} / \mathrm{kg}$ over five minutes. Then propofol infusion was started at $50 \mathrm{mcg} / \mathrm{kg} / \mathrm{min}$ to achieve bis value 50-60. Then endoscopy was done. If the subject did not tolerate the endoscope or patient experienced pain during the entire procedure then additional propofol bolus of $0.3 \mathrm{mg} / \mathrm{kg}$ was given. Similarly in PD group, the subject was given $1 \mathrm{mcg} / \mathrm{kg}$ dexmeditomidine instead of Fentanyl, rest the same. Meanwhile HR, BIS value, SPO2, MAP were noted. Results: It was found that there was significant difference in $\mathrm{SpO} 2$ Heart rate,,Mean, BIS Meanwhile $54.3 \%$ of patients required airway manoeuvre to maintain Saturation in PF group while only $2.9 \%$ patients of PD group required airway support. This difference in airway manoeuvre was statistically significant. Conclusion: we concluded that propofol dexmeditomidine group had better respiratory parameters, better hemodynamic stability, lesser need of total propofol. Propofol dexmeditomidine had better satisfaction levels among patients as compared to propofol fentanyl group butrecovery time of Propofol-fentanyl group was faster than propofol dexmeditomidine group.Except for time taken for recovery, PD group was both safer and more efficacious. Overall Propofol Dexmeditomidine group achieved better conditions for sedation in upper GI endoscopy than Propofol-Fentanyl.

Keywords: Fentenyl, Dexmeditomedine, BIS monitoring, Endoscopy.

Corresponding Author: Dr Sandeep Killedar, Senior Resident Department of Anaesthesiology and Critical care, Sapthagiri Institute of Medical Sciences \& Research Centre, Chikkasandra, Hesaraghatta Main Road, Bangalore - 560090

Received: July 2019

Accepted: July 2019

Introduction

Endoscopy in patients with gastrointestinal disorders (GI) is of immense benefit for diagnostic and therapeutic measures. Inspite of use of flexible fibreoptic equipments, endoscopy remains an unpleasant experience for most patients. The purpose of sedation in these patients is to relieve anxiety, discomfort or pain, and diminish memory of the event. ${ }^{[1]}$ There has been a general consensus that moderate sedation provides adequate control of pain and anxiety during endoscopic procedures. Conscious sedation enables patients to maintain their response to verbal and tactile stimuli without losing cardiovascular and ventilatory function. ${ }^{[2]}$ The anaesthetic drugs that are usually used include benzodiazepines, ketamine, fentanyl, propofol, dexmeditomidine etc. Each class of anaesthetic drugs has a different combination of anxiolytic, hypnotic, amnestic, and analgesic effects. Selection of the most appropriate medication for a specific patient requires consideration of many factors such as potential drug interactions, pharmacokinetics and pharmacodynamics of each drug. ${ }^{[3]}$ The ideal sedative is free of serious adverse effects; is not associated with significant drug interactions; does not accumulate with repeated dosing even in the presence of 
organ dysfunction; is easy to administer; has a quick and predictable onset and dissipation of effect and is inexpensive. ${ }^{[3]}$

It is known that combining the two agents for sedation and analgesia for outpatient procedures may preserve sedation efficacy while minimising respective adverse effects. ${ }^{[4]}$

\section{Subjects and Methods}

This prospective randomised comparative study was conducted in BGS Global Hospitals,Bangalore After getting the institutional Ethical commitee clearance .After written informed consent 70 patients of either sex, aged between 20 and 60 years who belong to ASA physical status I, II and III scheduled for elective upper GI endoscopies under sedation lasting atleast for $30 \mathrm{~min}$ were included in this study. Emergency, ASA Grade III \& IV, Patients requiring intubation for the procedure, difficult airway patients where excluded. The study group was divided in two groups of 35 each, Group A Propofol-Fentanyl (PF) and Group (B) Propofol-Dexmeditomedine (PD).

Preanaesthetic evaluation, premedication, standard monitors connected Standard anaesthesia protocol was followed. All patients were shifted to the procedure table A multiparameter monitor was attached: 3 lead ECG, Pulse Oximetry, NIBP, HR , BIS were recorded preinduction and postinduction of anaesthesia was done as per hospital protocol.

Group A Propofol-Fentanyl (PF) patient was administered fentanyl $2 \mathrm{mcg} / \mathrm{kg}$ initially followed by Propofol loading dose of $1.5 \mathrm{mg} / \mathrm{kg}$ over five minutes. Then propofol infusion was started at $50 \mathrm{mcg} / \mathrm{kg} / \mathrm{min}$ to achieve bis value $50-60$. Then endoscopy was done. If the subject did not tolerate the endoscope or patient experienced pain during the entire procedure then additional propofol bolus of $0.3 \mathrm{mg} / \mathrm{kg}$ was given. Similarly in Group (B) Propofol-Dexmeditomedine (PD) group, the subject was given $1 \mathrm{mcg} / \mathrm{kg}$ dexmeditomidine instead of Fentanyl, rest the same. Meanwhile HR, BIS value, SPO2, MAP were noted.

If any airway manoeuvre /intervention required maintaining haemoglobin oxygen saturation was noted in both groups. At the completion of the procedure, background infusion of the Propofol was stopped and BIS value allowed equilibrating above 80 . Patients oropharynx thoroughly suctioned, turned supine with head up tilt (15 degrees), allowed for complete recovery with end points being eye opening on command, ability to handle secretions, follow simple commands, hemodynamic stability, maintaining room air saturation $>95 \%$ and attainment of BIS value >90. Recovery characteristics were noted using Modified Aldrete Score.

\section{Results}

Seventy patients under sedetion were studied. Age of the patient varied from 20 to 70 years. Mean age in years of Group A was 46 \pm 12.2 and Group B was $46.4 \pm 12.4$

In Group A, $42.9 \%$ had SpO2 $<94$ once and $2.9 \%$ had $\mathrm{SpO} 2$ $<94 \%$ twice, were as in Group B, SpO2 was $<94 \%$ once in $2.9 \%$ and none had twice. This difference in $\mathrm{SpO} 2<94 \%$ between two groups was statistically significant.

Mean $\mathrm{SpO} 2$ was significantly lower in Group $\mathrm{A}(\mathrm{PF})$ compared to Group B(PD).

In the study there was no significant difference in mean Heart rate between two groups [Figure 2]

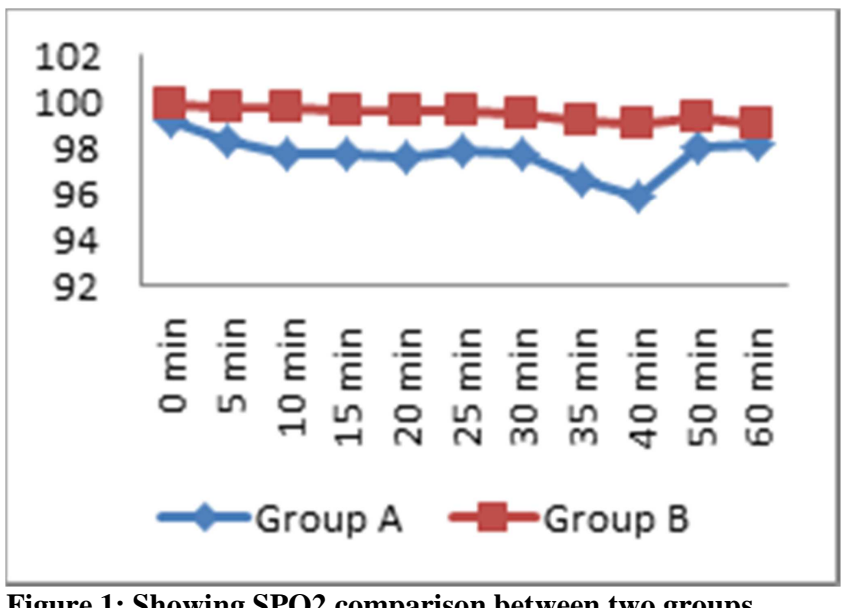

Figure 1: Showing SPO2 comparison between two groups

$\chi 2=17.49, \mathrm{df}=2, \mathrm{p}<0.001 *$

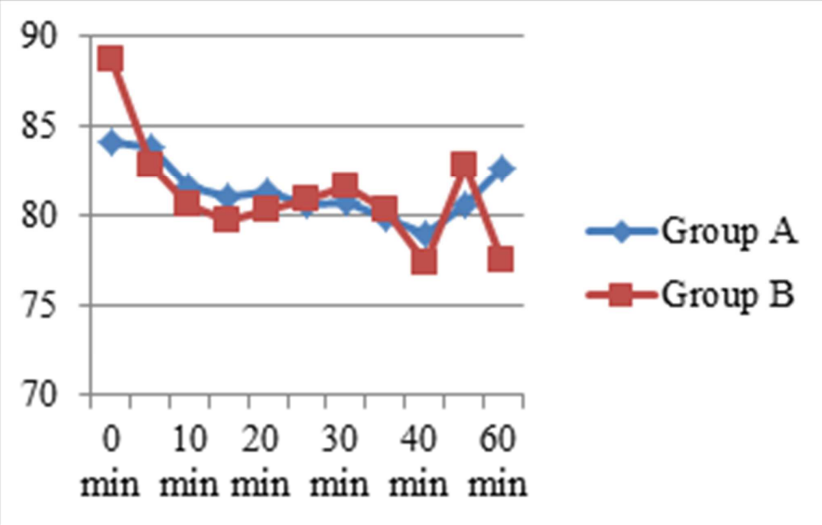

Figure 2: Showing Heart rate comparison between two groups

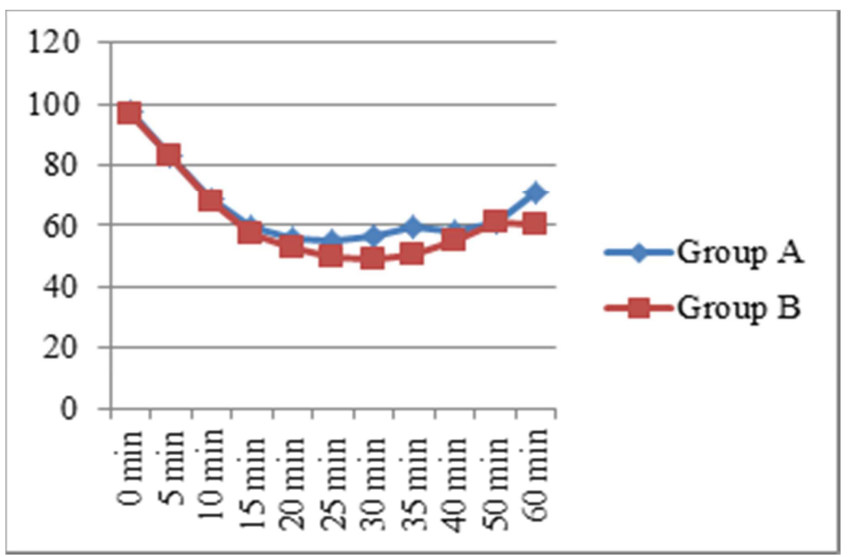

Figure 3: Showing BIS comparison between two groups

In the study there was significant difference in BIS score between two groups at $25 \mathrm{~min}, 30 \mathrm{~min}$ and $35 \mathrm{~min}$ of follow up. BIS was higher in Group A compared to Group B at these intervals.

In Group A mean of Lowest BIS was $42.9 \pm 9.7$ and in Group B was $36.6 \pm 7.7$. This difference in mean Lowest BIS 
was statistically significant.

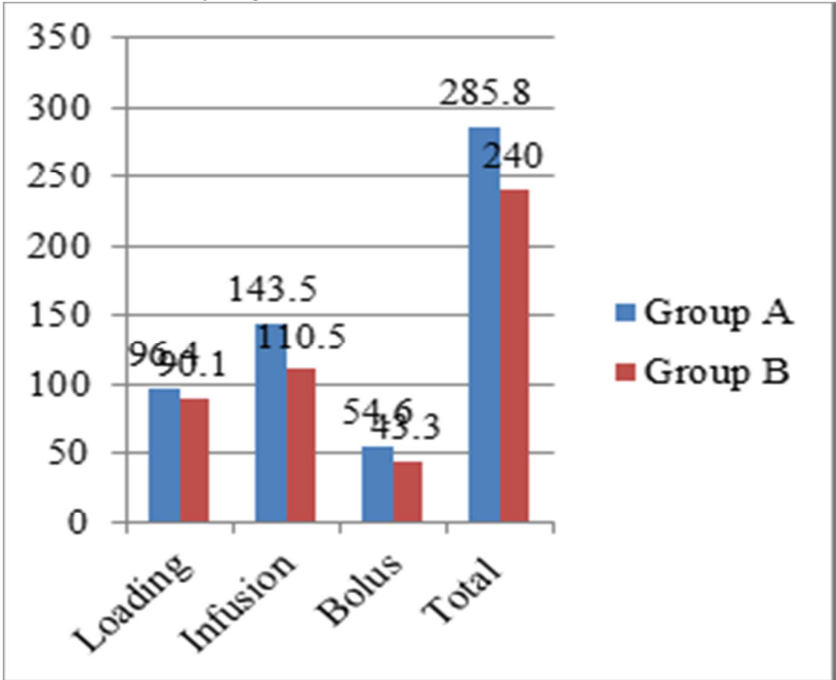

Figure 4: Showing Propofol used comparison between two groups

Mean Propofol used at Loading, Infusion, Bolus and total was higher in Group A than in group B. Significant difference in Propofol used was observed at Infusion and Total dose

In the Group A $45.7 \%$ of subjects did not require Airway manoeuvre, in Group B $97.1 \%$ did not require airway intervention. This difference in airway manoeuvre was statistically significant $(\chi 2=23.05, \mathrm{df}=4, \mathrm{p}<0.001 *)$.

\section{Discussion}

Sedation for upper GI endoscopies should have rapid onset and short duration of action. An adequate depth of sedation and analgesia is required to alleviate patient's discomfort. Sedation in endoscopy is more complex than other procedural sedation due to sharing of the upper airway and positioning of the patient in left lateral or semi prone position.

Sedation with Dexmedetomidine in upper GI endoscopy is promising with increased patient satisfaction, maintenance of natural sleep cycle and bettertolerance, including turning and suctioning. ${ }^{[12,14]}$ It also has anaesthetic and opioid sparing effect in general anaesthesia when used as an adjuvant12. The most important aspect of sedation with Dexmedetomidine is the quality of the cooperative sedation. Patients display a unique arousability, positive respiratory profile with the maintenance of adequate spontaneous respiration and patency of the upper airway and appropriate ventilatory response to hypoxia and hypercarbia. ${ }^{[15]}$

In our study, there was significant difference in mean $\mathrm{SpO} 2$ between two groups at all the intervals of follow-up, except at $40 \mathrm{~min}$ and $60 \mathrm{~min}$. Mean $\mathrm{SpO} 2$ was significantly lower in Group PF compared to Group PD.

In our study we found that airway manoeuvres were used more in PF than PD group. In PF group $45.1 \%$ did not require any airway manoeuvrei.e $55 \%$ requiring airway support whereas in PD group $97.1 \%$ did not require any airway manoeuvre i.e. only $2.9 \%$ requiring airway support. In our study PD group had better respiratoryparameters overall.

We found that propofol consumption in PD group was lower than PF group. There was significant difference in Propofol used which was observed at Infusion and Total dose. In PD group,there was $23 \%$ reduction of propofol at induction and $15.78 \%$ reduction in total propofol consumed when compared to PF group.

There was significant difference in BIS score between two groups at $25 \mathrm{~min}, 30 \mathrm{~min}$ and $35 \mathrm{~min}$ of follow up. BIS was higher in Group PF compared to Group PD at these intervals. We were able to conduct our study with Bis value of 50-60 in both groups.

\section{Conclusion}

From our study we concluded that propofol dexmeditomidine group had better respiratory parameters, better hemodynamic stability, lesser need of total propofol. Propofol dexmeditomidine had better satisfaction levels among patients as compared to propofol fentanyl group butrecovery time of Propofol-fentanyl group was faster than propofol dexmeditomidine group. Except for time taken for recovery, PD group was both safer and more efficacious. OverallPropofol Dexmeditomidine group achieved better conditions for sedation in upper GI endoscopy than PropofolFentanyl.

Propofol dexmeditomidine can be effectively used for sedation as an alternative to propofol fentanyl

\section{References}

1. Nagaraj M, Geetha C, Rajavardhan. Is dexmeditomidine poor surrogate to propofol for procedural sedation during endoscopic retrograde cholangiopancretography (ERCP).JEMDS 2013 October;(2):8165-75

2. David $\mathrm{R}$ Lichenstein et al published 'Guideline for Sedation and anesthesia in GI endoscopy'in the American Society of Gastrointenstinal Endoscopy Journal.November2008 Volume 68, Issue 5, Pages 815-826.

3. MohanadShukrey et al Update on dexmedetomidine: use in nonintubated patients requiring sedation for surgical procedures.Ther and Clin Risk Manag.April 2010; Volume 6:111-121

4. Sanjay Arora. Combining Ketamine and Propofol(Ketopol) for emergency department procedural sedation and analgesia. West JEM.2008;9:20-23.

5. Abeles $\mathrm{G}$ et al.The combined use of propofol and fentanyl for outpatient intravenous conscious sedation.Dermatolog surg. 1999 Jul;25(7):559-62

6. Sandeep Dutta et al. Effect of dexmedetomidine on propofol requirements in healthy subjects.JPharmSci.February 2001; Volume 90, Issue 2, Pages 172-181

7. Sivak MV. Endoscopic technology:is this as good as it gets?1999;50:718-719

8. Kawahara I. Flexible endoscopic technology: the fibreopticendo scope. In: Gastroenterologic Endoscopy,2000:2nd Edn16-28.

9. Trummel Jm. Comparison of differing sedation practice for upper endoscopic ultrasound using expert observational analysis of the procedural sedation.J Patient Safety 2009 Sep;5(3):153-9.

10. Benjamin Tharian, Nayana George et al. Endoscopic ultrasound fine needle aspiration: Technique and applications in clinical practice.World J GastrointestEndosc. 2012 Dec 16; 4(12): 532-544.

11. Bryson HM, Foulton BR, Faulds D. Propofol:an update of its use in anesthesia and conscious sedation.Drugs. 1995;50:513-559

12. Elbaradie S, Mahalawy FH, and Solyman AH. Dexmedetomidine vs. propofol for short-term sedation of postoperative mechanically ventilated patients. Journal of the Egyptian nat. cancer inst 2004 September; 16(3):153-158.

13. Herr DL, Sum-Ping STJ, England M. ICU sedation after coronary artery 
bypass graft surgery: dexmedetomidine-based versus propofol-based sedation regimens. Journal of cardiothoracic and vascular anesthesia 2003 October; 17(5):576-584.

14. Bekker A, Sturaitis MK. Dexmedetomidine for neurological surgery.
Operative Neurosurgery 2005 July; 57(1):1-10.

15. Kruger P. Dexmedetomidine in sedation. S Afr J Anaesthesiol Analg 2010;16(1):102-104.

Copyright: (ㅇ the author(s), publisher. Academia Anesthesiologica International is an Official Publication of "Society for Health Care \& Research Development". It is an open-access article distributed under the terms of the Creative Commons Attribution Non-Commercial License, which permits unrestricted non-commercial use, distribution, and reproduction in any medium, provided the original work is properly cited.

How to cite this article: Basarigidad A, Killedar S. Comparative Study of Safety and Efficacy between Propofol-Fentanyl Versus PropofolDexmeditomidine Combination For Sedation in Upper Gastro-Intestinal (GI) Endoscopic Procedures- A Prospective Randomised Study. Acad. Anesthesiol. Int. 2019;4(2):67-70.

DOI: dx.doi.org/10.21276/aan.2019.4.2.18

Source of Support: Nil, Conflict of Interest: None declared. 